\title{
La radioprotection dans les mines et usines de minerai d'uranium françaises
}

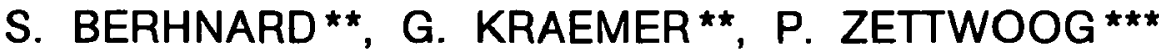

(Manuscrit reçu le 28 octobre 1988)

RÉSUMÉ Une nouvelle réglementation concernant la radioprotection des travailleurs dans les mines a été récemment introduite dans le code minier français. Elle prend en compte les recommandations de la Commission internationale de protection radiologique (CIPR), publication 26, et les directives européennes de 1980 et 1984 . Cette nouvelle réglementation est appliquée avec succès depuis 1983 par les exploitants d'uranium français.

Pour la surveillance dosimétrique des mineurs d'uranium, on utilise un dosimètre individuel permettant de mesurer les trois équivalents de dose mensuels (exposition externe, exposition interne due à l'énergie alpha potentielle des descendants du radon 222, exposition interne due aux émetteurs alpha à vie longue). La prévention des risques radiologiques est basée sur la détection rapide des défaillances de la ventilation primaire et secondaire par des contrôles réguliers des ambiances physiques.

ABSTRACT A regulation concerning workers' radiation protection in mines has been recently introduced in the french "Code Minier"; it takes into account the ICPR recommendations of Publication 26 and the European directives of 1980 and 1984. This new regulation is being implemented with success in France since 1983.

For workers' monitoring in uranium underground mines, an individual dosimeter measuring the three monthly dose equivalents (gamma, potential alpha energy, long-lived alpha emitters) is compulsory. The aim of the ambient monitoring is the early prevention of every defects in the primary and auxilliary ventilation, which is the basis of prevention.

\section{INTRODUCTION}

Lorsque, peu avant 1950, furent ouvertes les premières mines d'uranium en France, la nature exacte des risques radiologiques auxquels allaient être exposés les mineurs était mal connue, en particulier en ce qui concerne le radon 222 . Les premières recherches effectuées pour apprécier l'importance du radon datent de 1953. Cette année-là étaient exécutés les premiers travaux d'exploitation sur le gisement des Bois Noirs, dans les Monts du Forez et la première centaine de tonnes d'uranium était produite en France. Dès 1955, les premiers éléments

* CRPM/Fanay, 87240 St-Sylvestre.

** Cogéma/BM, 2, rue Paul Dautier, 78141 Vélizy-Villacoublay.

*** Commissariat à l'énergie atomique, IPSN, BP 6, 92265 Fontenay-aux-Roses Cedex. 
d'une doctrine de la prévention des risques radiologiques miniers et de la surveillance dosimétrique étaient disponibles et, à partir de 1956, l'établissement de fiches individuelles dosimétriques était effectif pour tous les mineurs [8].

Ainsi, depuis plus de trente ans, la politique française en matière de radioprotection dans les mines se poursuit avec une remarquable continuité, tant en ce qui concerne les objectifs que son organisation.

\section{IDENTIFICATION DES RISOUES RADIOLOGIOUES}

Les mineurs d'uranium reçoivent l'essentiel de leur exposition aux rayonnements au cours des opérations de routine liées à l'extraction du minerai, les opérations de maintenance normalement effectuées dans des zones stériles étant relativement moins irradiantes. Dans le cycle du combustible nucléaire, cette première étape constituée par l'exploitation du minerai d'uranium est celle où la dose individuelle moyenne est la plus élevée : en effet, il n'en est guère ainsi dans les autres étapes du cycle que pour les personnels chargés de la maintenance, de la décontamination et des tâches de démantèlement. La particularité qu'ils ne partagent avec aucune autre catégorie de travailleurs est que leur exposition, d'origine naturelle, se compose en routine non seulement d'une exposition externe mais aussi d'une exposition interne d'importance semblable.

Dans une mine d'uranium, les mineurs se trouvent exposés aux rayonnements issus de l'ensemble des six radionucléides émetteurs alpha et des huit radionucléides émetteurs bêta de la chaîne de l'uranium 238. Les radionucléides présents dans le minerai, en place ou abattu, sont d'abord une source de rayonnements gamma, $83 \%$ du rayonnement en énergie venant du bismuth $214,12 \%$ du plomb 214 , tous deux descendants à vie courte du radon 222 . Typiquement, au centre d'une galerie tracée dans du minerai à une teneur en uranium de $0,1 \%$, le débit de dose est de l'ordre de $5 \mu \mathrm{Gy} . \mathrm{h}^{-1}$ et le risque de dépassement de la limite annuelle de $50 \mathrm{mSv}$ pour l'organisme entier existe dès que la teneur du minerai dépasse $0,5 \%$. II n'est pas rare actuellement de mesurer dans des chantiers en cours d'exploitation des débits de dose ambiants compris entre 50 et $200 \mu \mathrm{Gy} \cdot \mathrm{h}^{-1}$.

Un second type de risque radiologique encouru par les mineurs est caractérisé par une contamination interne provenant de l'inhalation des émetteurs alpha en suspension dans l'atmosphère:

- le radon 222 et ses produits de filiation, émetteurs alpha à vie courte ;

- les poussières de minerai, émetteurs alpha à vie longue.

Parmi les descendants de l'uranium 238 se trouve le radon 222, gaz rare susceptible de migrer dans les roches et de polluer l'atmosphère. L'inhalation du radon 222 lui-même, bien qu'il engendre des descendants radioactífs à vie courte dans les poumons ainsi que dans les autres tissus de l'organisme après une faible dissolution dans le sang, n'entraîne, en 
général, qu'un risque négligeable devant celui qui découle de l'inhalation de ces mêmes descendants formés dans l'air. Les polonium 218, plomb 214 , bismuth 214 , soit à l'état d'ions libres, soit fixés sur les aérosols miniers, se déposent, en effet, dans les poumons et y laissent une large part de leur énergie potentielle de désintégration. L'exposition des mineurs à des concentrations élevées de radon 222 et de ses descendants a été correlée avec l'induction de cancers du poumon dans plusieurs groupes de mineurs (mine de la région de Schneeberg en Allemagne ou de Joachimstal en Tchécoslovaquie) et ces constatations ont été renforcées par la reproduction expérimentale de cancers bronchopulmonaires chez des rats exposés uniquement à des inhalations de radon 222 et de ses descendants.

Le second risque de contamination interne est provoqué par la formation de poussières fines de minerai, chargées d'éléments de la chaîne de l'uranium, émetteurs alpha à vie longue : ${ }^{238} \mathrm{U},{ }^{234} \mathrm{U},{ }^{230} \mathrm{Th},{ }^{226} \mathrm{Ra},{ }^{210} \mathrm{Po}$. Les atomes inhalés sont susceptibles de se déposer dans les poumons, mais la plus grande partie est éliminée biologiquement avant d'avoir pu se désintégrer et délivrer leur énergie. Cependant, le risque de contamination peut devenir critique vis-à-vis des limites annuelles dans des chantiers fortement empoussiérés et où la teneur du minerai dépasse $0,5 \%$. Ce risque a toujours été pris en compte en France, avec juste raison, car il n'est pas toujours négligeable et peut même devenir prépondérant dans certains cas comme dans les mines à ciel ouvert dans les régions à climat sec.

Le risque "poussières" est prépondérant également dans les usines de traitement du minerai. Dans ces installations, on retrouve les deux types de risques d'irradiation interne et externe décrits pour les mines, avec quelques différences selon la phase du traitement dans laquelle travaille le personnel: dans la zone de préparation mécanique du minerai, les risques sont semblables à ceux rencontrés lors des opérations d'extraction. Dans les zones d'attaque chimique du minerai, les risques d'irradiation interne sont très faibles ; ils réapparaissent dans la zone de finition du produit avec, essentiellement, les problèmes liés aux poussières d'uranate. Contrairement aux mines où les risques radiologiques sont inhérents aux opérations d'extraction elles-mêmes, dans les usines de traitement du minerai, il se rapportent principalement aux opérations d'entretien et de maintenance du matériel de production. Ainsi, l'expérience a montré que le risque d'exposition du personnel des usines à des doses élevées en des temps courts était réel si des dispositions particulières n'étaient pas prises avant les interventions, lors de l'entretien de cuves d'attaque ou des colonnes de résines notamment.

Le risque d'exposition à des doses relativement élevées durant des périodes limitées dans le temps peut également être présent dans les mines souterraines : il suffit que la ventilation ne soit plus assurée dans un chantier pour que, en quelques heures, on atteigne des concentrations en énergie alpha potentielle due aux descendants à vie courte du radon 222 de l'ordre de 100 à $1000 \mu \mathrm{J} . \mathrm{m}^{-3}$, soit environ 10 à 100 fois la valeur guide opérationnelle dans l'air. 
La figure 1 présente l'importance relative des différents risques dans la dose moyenne annuelle (représentant le cumul des doses de différentes natures) pour l'ensemble des installations' d'extraction et de traitement en France en 1989.

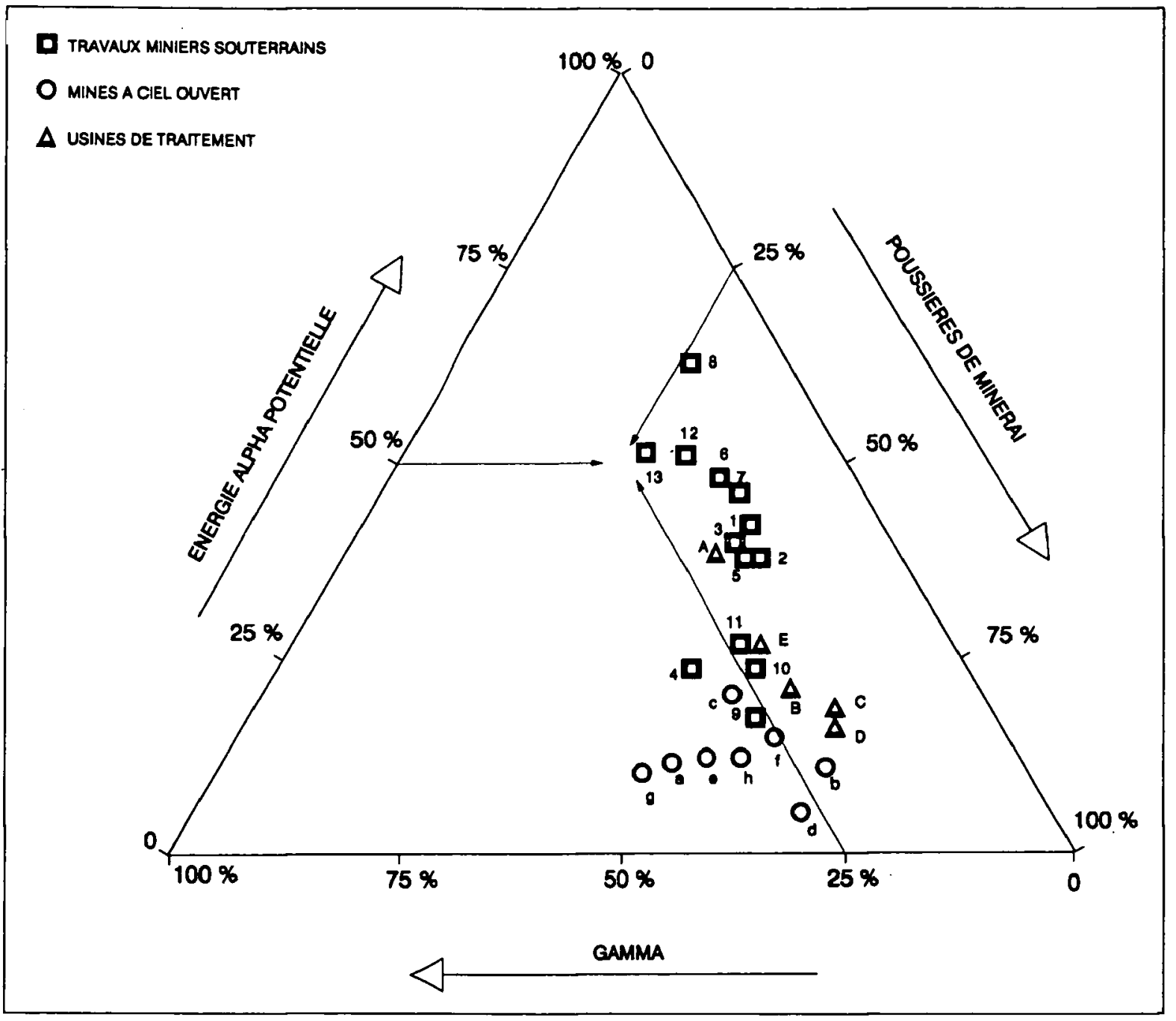

Fig. 1. - Importance de chaque risque dans le cumul des expositions pour les exploitations françaises en 1989

\section{LA RÉGLEMENTATION}

Les objectifs de la radioprotection en France ont toujours été l'amélioration constante de la qualité de la surveillance des risques individuels et collectifs et de l'efficacité de leur prévention. Cette amélioration a été le fruit d'une collaboration étroite entre les responsables des techniques de protection dans les activités minières, les experts français des grandes institutions internationales compétentes comme la Commission internationale de protection radiologique (CIPR) ou l'Euratom et les rédacteurs des textes réglementaires qui conseillent les pouvoirs publics. 
Il en a été ainsi dès les années 1955-1956 pour l'instauration d'un suivi dosimétrique individuel de chaque mineur prenant en compte le cumul des trois risques radiologiques auxquels ils sont exposés : l'exposition externe et les expositions internes dues au radon 222 et aux poussières de minerai.

Une première réglementation voit le jour en 1958. Elle est en partie reprise et complétée en 1965 sous la forme d'un arrêté préfectoral type réglementant les travaux d'exploitation et de recherches de substances radioactives dans les départements concernés (circulaire ministérielle $\mathrm{DM} / \mathrm{H} \mathrm{n}^{\circ} 119$ du 4 mars 1965).

Au Journal Officiel du 20 juillet 1989 a été publié, sous la signature du Premier ministre et du ministre de l'industrie et de l'aménagement du territoire, un décret ( $n^{\circ} 89-502$ du 13 juillet 1989) qui introduit, dans le Règlement général des industries extractives (RGIE), un titre intitulé "Rayonnements ionisants" dont la première partie, relative à la protection du personnel, fait l'objet d'une annexe au décret. Dans le même numéro du Journal Officiel, une circulaire du ministre de l'industrie aux préfets présente le décret et en commente, en annexe, les différentes prescriptions. Le champ d'application de ce décret porte non seulement sur la recherche et l'exploitation de substances radioactives mais également sur la surveillance de l'atmosphère des travaux souterrains des autres substances.

Pour les usines de traitement non soumises au RGIE s'applique, depuis octobre 1987 , le décret $n^{\circ} 86-1103$ du 2 octobre 1986 relatif à la protection des travailleurs contre les dangers des rayonnements ionisants.

Dans les deux textes applicables aux mines et aux usines, on retrouve un grand nombre de similitudes, en particulier en ce qui concerne les principes généraux de protection avec l'introduction, pour le risque collectif, du principe d'optimisation développé par la CIPR et les limites du cumul des équivalents de dose individuels.

Indépendamment de la mise à jour des limites annuelles et trimestrielles concernant les différentes modalités d'exposition aux rayonnements ionisants, l'une des caractéristiques techniques essentielles de la nouvelle réglementation est que la surveillance dosimétrique du personnel d'une part, et la surveillance des ambiances radioactives des postes de travail d'autre part, sont traitées selon des procédures et des matériels spécifiques. Dans les paragraphes suivants seront présentés ces procédures et matériels qui, bien que nouvellement imposés par la réglementation, sont en place dans les mines et les usines françaises depuis 1983. En effet, depuis plusieurs années, la politique en matière de radioprotection suivie par les industriels français et les autorités de tutelle a pris en compte les principes de la CIPR afin d'éviter une discontinuité, dans la pratique et dans les résultats, de la surveillance radiologique des exploitations, liée à l'application de la nouvelle réglementation plus sévère que la précédente. Ainsi, il apparaît, dès la fin de 1986, grâce 
aux efforts entrepris, que les nouvelles limites individuelles peuvent être respectées par la quasi-totalité des travailleurs, ce qui n'aurait pas été le cas en 1983.

\section{LIMITES ET TAUX D'EXPOSITION}

Le décret $n^{\circ}$ 88-521 du 18 avril 1988 modifiant celui du 20 juin 1966 relatif aux principes généraux de protection contre les rayonnements ionisants définit, pour les travailleurs, les limites qui doivent impérativement être respectées pour chaque modalité d'exposition considérée séparément. Ces limites sont reprises sous la forme de limites annuelles dans le décret $n^{\circ}$ 89-502 pour les différentes modalités qui concernent le personnel des industries extractives:

- pour l'exposition externe, la limite annuelle d'équivalent de dose maximal en profondeur est de $50 \mathrm{mSv}$;

- pour l'exposition interne entraînée par l'inhalation des descendants à vie courte du radon, la limite annuelle d'énergie alpha potentielle inhalée est de $20 \mathrm{~mJ}$ pour le radon 222 et de $60 \mathrm{~mJ}$ pour le radon 220 ;

- pour l'exposition interne entraînée par l'inhalation des poussières de minerai, la limite annuelle d'activité alpha des radionucléides à vie longue inhalés est de $1700 \mathrm{~Bq}$ pour les poussières de minerai d'uranium ${ }^{1)}$, et de $300 \mathrm{~Bq}$ pour les poussières de minerai de thorium $232^{1)}$;

- pour l'exposition interne entraînée par l'inhalation des poussières d'uranate, la limite annuelle d'activité alpha des radionucléides à vie longue inhalés est de $30000 \mathrm{~Bq}$ avec la restriction que la quantité pondérale journalière inhalée n'excède pas $2,5 \mathrm{mg}$ pour des raisons de toxicité chimique.

En vue de faciliter les opérations de cumul des risques définies dans l'annexe IV du décret $n^{\circ} 88-521$ du 18 avril 1988, la législation adopte ici une terminologie simplificative. Pour chaque modalité d'exposition, le "taux d'exposition pendant une durée déterminée ( 3 mois ou 12 mois consécutifs par exemple)" est défini comme le rapport de l'exposition subie pendant cette durée à la limite annuelle correspondante. Pour toutes les modalités d'exposition auxquelles sont soumis les travailleurs, le "taux d'exposition totale" sera la somme des taux

1) Ces valeurs sont celles qui ont été calculées par la CIPR, Publication 47 "Radiation protection of workers in mines", 1985. 
partiels relatifs à chacune de ces modalités. On aura ainsi, en général, pour les mineurs et agents des usines:

$$
\begin{aligned}
\text { TET }=\frac{H}{50 \mathrm{mSv}}+ & \frac{\mathrm{E} \alpha \text { Pot. }{ }^{222} \mathrm{Rn}}{20 \mathrm{~mJ}}+\frac{A \alpha \text { Pous. minerai }}{1700 \mathrm{~Bq}}+ \\
& \frac{\text { A } \alpha \text { Pous. uranate }}{30000 \mathrm{~Bq}}
\end{aligned}
$$

avec

TET = taux d'exposition totale,

$\mathrm{H}$ = équivalent de dose maximal en profondeur,

$\mathrm{E} \alpha$ Pot. ${ }^{222} \mathrm{Rn}=$ énergie alpha potentielle des descendants à vie courte du radon 222 inhalée,

A $\alpha$ Pous. minerai $=$ activité alpha des radionucléides à vie longue des poussières de minerai inhalées,

$A \propto$ Pous. uranate $=$ activité alpha des radionucléides à vie longue des poussières d'uranate inhalées.

Les limites générales d'exposition pourront alors s'exprimer d'une façon simple: le taux d'exposition totale pour un agent ne doit pas être supérieur à 1 pour 12 mois consécutifs, à 0,6 pour 3 mois consécutifs, avec la contrainte de toxicité chimique: masse d'uranate inhalée en 1 jour $<2,5 \mathrm{mg}$.

Dans le cas de mines de substances radioactives particulièrement difficiles, lorsque les moyens normaux de lutte contre les rayonnements ionisants s'avèrent insuffisants, la nouvellè réglementation prévoit que, sur autorisation du préfet et en fonction d'un certain nombre de conditions, la valeur maximale du taux annuel d'exposition totale puisse être respectée en moyenne sur une durée de cinq ans pour tout ou partie du personnel, sans toutefois dépasser 1,5 sur 12 mois consécutifs et 0,9 sur 3 mois consécutifs.

En ce qui concerne les mines souterraines non uranifères, le décret $n^{\circ}$ 89-502 du 13 juillet 1989 complétant le règlement général des industries extractives précise, dans sa section 5 , article 46 , que la présence de radon et de ses descendants doit être recherchée au début des travaux puis tous les trois ans, au moins sur les ouvrages de retours d'air qui aboutissent en surface. Dans le cas où le personnel de l'exploitation est susceptible de dépasser 0,1 pour le taux annuel d'exposition, les dispositions des chapitres II et III de la section 1 (surveillance du personnel et dosimétrie) sont applicables. La circulaire d'application précise que si l'activité alpha volumique du radon 222 est supérieure à $400 \mathrm{~Bq} \cdot \mathrm{m}^{-3}$ sur les retours d'air, il y a lieu d'entreprendre une étude plus approfondie du champ d'exploitation. La procédure d'identification des mines à risque radon par mesure sur leurs retours d'air et les résultats obtenus sur un certain nombre de mines non uranifères entre 1980 et 1984 sont décrits en [1] et [2]. 


\section{SURVEILLANCE DOSIMÉTRIQUE DES TRAVAILLEURS}

\subsection{Stratégie de dosimétrie}

Trois types de dosimétrie peuvent être utilisés pour connaître la situation dosimétrique des travailleurs:

- une dosimétrie individuelle basée sur l'utilisation d'appareils portés par les agents durant leur poste de travail et permettant de mesurer en continu sur un mois les expositions interne et externe subies par chaque travailleur;

- une dosimétrie de fonction basée sur l'utilisation d'appareils portés par un échantillon de personnes représentatif des différentes fonctions que l'on peut rencontrer dans l'exploitation concernée ; ces fonctions sont telles que les expositions susceptibles d'être reçues sont considérées comme de même nature et de même intensité ; les appareils permettent de déterminer les niveaux moyens d'exposition interne et externe pour chacune des fonctions et à partir des temps de travail effectif des agents dans les fonctions, on peut calculer les expositions subies par chacun d'eux ;

- une dosimétrie d'ambiance basée sur des contrôles de la qualité de l'atmosphère des lieux de travail et au cours des différentes phases de travail (par exemple : contrôles des concentrations en radon 222 ou en poussières de minerai ou contrôle des niveaux d'énergie alpha potentielle due aux descendants du radon 222). A partir de ces mesures, on peut calculer les concentrations moyennes en radionucléides dans chaque lieu de travail et, en fonction du temps passé par les agents dans ces lieux, on détermine les expositions subies par chaque travailleur.

Le décret $n^{\circ} 89-502$ du 13 juillet 1989 complétant le RGIE (article 13) préconise de mettre en place:

- une dosimétrie individuelle pour les personnes de catégorie A (taux annuel d'exposition totale susceptible d'être supérieur à 0,3 ) ;

- une dosimétrie individuelle ou de fonction pour les personnes de catégorie B (taux annuel d'exposition totale susceptible de ne pas dépasser 0,3 ).

Cette réglementation ne considère pas la possibilité de faire une dosimétrie d'ambiance.

Les agents susceptibles de subir un taux annuel d'exposition totale inférieur au $1 / 10^{\mathrm{e}}$ de la limite annuelle sont considérés comme non exposés aux rayonnements ionisants et ne sont pas contrôlés en dosimétrie.

Ce sont ces dispositions qui sont appliquées pour la surveillance radiologique des mineurs d'uranium français depuis 1983, soit:

- une dosimétrie individuelle pour la surveillance des agents travaillant dans les mines souterraines,

- une dosimétrie de fonction pour la surveillance des agents travaillant dans les mines à ciel ouvert et dans les usines de traitement. 
Les rédacteurs du nouveau titre "rayonnements ionisants" du règlement général des industries extractives, promulgué au niveau du ministère de l'industrie, ont choisi de fonder la dosimétrie du personnel des mines sur l'usage d'un dosimètre individuel permettant de surveiller tous les risques, rayonnements gamma, énergie alpha potentielle due aux descendants à vie courte du radon 222, du radon 220 et des poussières de minerai.

Après dix années d'expérimentation, ce dosimètre multirisque, mis au point par le laboratoire d'instrumentation minière du CRPM $^{2)}$, est opérationnel depuis le $1^{\text {er }}$ janvier 1983 et, depuis, la surveillance dosimétrique des travailleurs des mines et des usines de traitement est basée sur l'utilisation de cet appareil. On trouvera, dans [3] et [10], le détail des considérations qui ont conduit à fonder la dosimétrie des exploitations françaises sur l'usage de ce dosimètre individuel multirisque.

\subsubsection{Description de l'appareil}

Porté pendant un mois par le travailleur, ce dosimètre mesure les expositions dues:

- aux aérosols radioactifs constitués des descendants à vie courte du radon 222 et du radon 220 , soit les isotopes 218,214 et 212 du polonium, émetteurs alpha;

- aux aérosols radioactifs constitués d'éléments à vie longue des chaînes de l'uranium et du thorium, émetteurs alpha ;

- aux rayonnements gamma ambiants.

Le dosimètre individuel intégré est un appareil pesant environ $300 \mathrm{~g}$ (dimensions : $85 \times 62 \times 79 \mathrm{~mm}$ ) composé d'un groupe de pompage centrifuge de débit nominal $5 \mathrm{I} \cdot \mathrm{h}^{-1}$, d'une alimentation par batterie pour la pompe (l'autonomie de la batterie est supérieure à $12 \mathrm{~h}$, la charge s'effectue par induction dès que l'appareil est placé dans son alvéole de charge), d'une tête de mesure constituée d'un corps en alliage léger contenant les détecteurs pour la mesure des expositions.

\subsubsection{Mesure des irradiations internes}

L'air prélevé par le dosimètre au niveau de la tête de mesure traverse un filtre membrane de 1,2 $\mu \mathrm{m}$ de porosité qui retient les aérosols présents dans l'air de la mine et, en particulier, les radionucléides émetteurs alpha. Les émetteurs alpha à vie courte, descendants du radon 222 et du radon 220 , se désintègrent sur le filtre : les alpha émis normalement à la surface du filtre traversent les collimateurs et sont ralentis sélectivement en fonction de leur énergie d'émission pour pouvoir venir impressionner les plages sensibles du film détecteur (nitrate de cellulose KODAK LR 115 type III).

2) CRPM : Centre de radioprotection dans les mines, création commune de·l'IPSN, Département de protection technique et de la Cogéma - branche uranium naturel. 
S. BERNHARD, G. KRAEMER, P. ZETTWOOG

3.2. Mise en œuvre d'un système individuel de dosimétrie intégré (SIDI) (fig. 2)
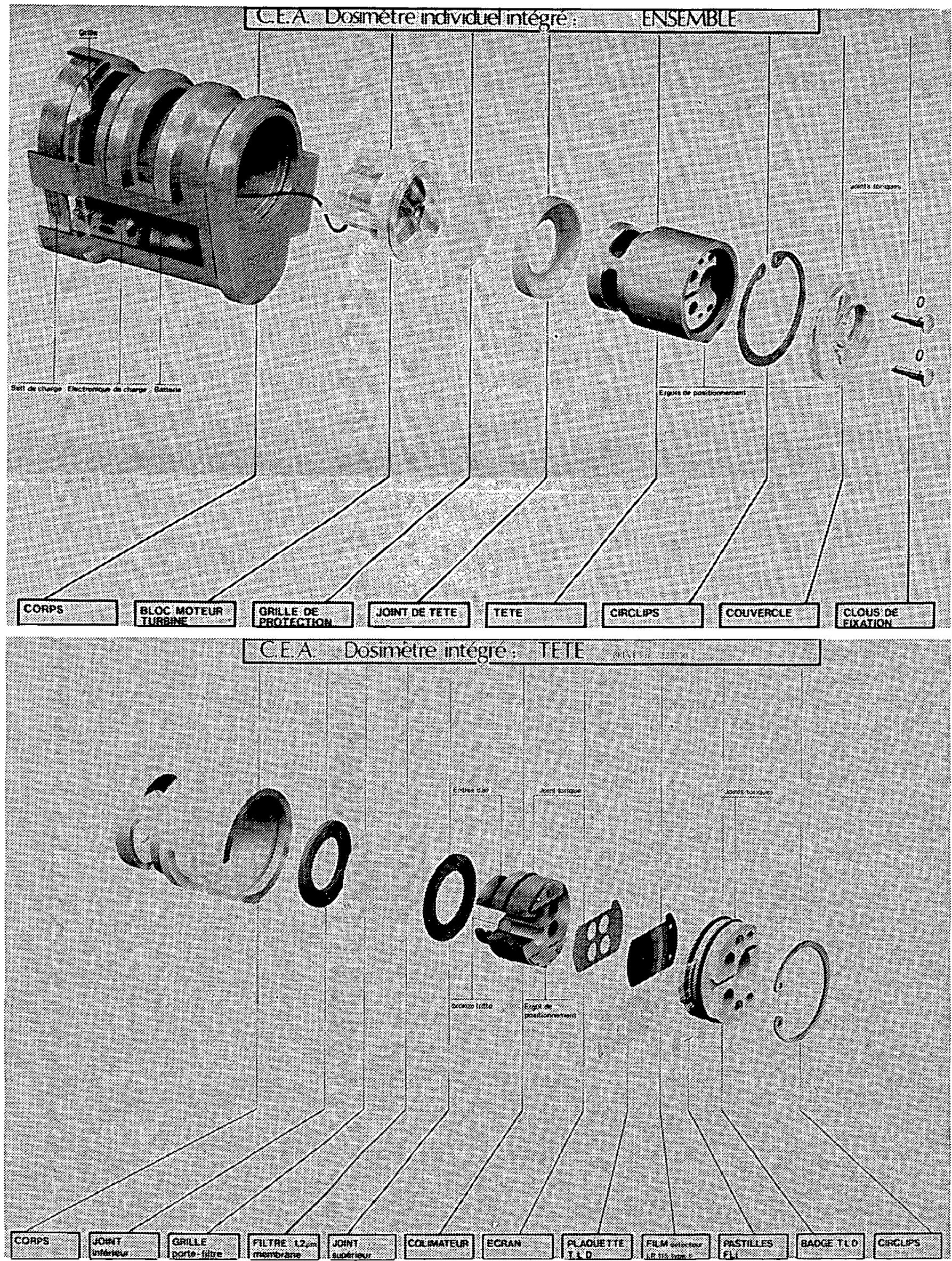

Fig. 2. 
Après un mois de port du dosimètre, la tête de mesure est envoyée au laboratoire d'exploitation dosimétrique:

- après développement dans un bain de soude, le nitrate de cellulose laisse apparaître sur 4 plages des traces correspondant aux émissions alpha des ${ }^{218} \mathrm{Po},{ }^{214} \mathrm{Po},{ }^{212} \mathrm{Bi},{ }^{212} \mathrm{Po}$ et ${ }^{222} \mathrm{Rn}$. Le nombre de traces enregistrées sur chacune des plages permet de calculer, en fonction du débit de prélèvement, l'exposition mensuelle à l'énergie alpha potentielle due aux descendants du radon 222 et du radon 220 reçue par le porteur du dosimètre ;

- au démontage de la tête, le filtre membrane de 1,2 $\mu \mathrm{m}$ est récupéré et on procède à un comptage alpha de ce filtre (avec photomultiplicateur et scintillateur) pour mesurer l'activité des aérosols émetteurs alpha à vie longue; en fonction du débit de prélèvement, on calcule l'exposition mensuelle du porteur du dosimètre aux poussières de minerai, seconde source d'irradiation interne.

On notera que le même filtre peut être utilisé pour la mesure de l'exposition à des toxiques non radioactifs, tels que les fibres de silice, les imbrûlés de fumées de moteurs diesel ou de tir, les métaux lourds. La faisabilité d'une mesure de l'exposition aux oxydes d'azote à l'aide de filtres imprégnés montés en série avec le filtre à poussières a également été démontrée.

\subsubsection{Alesure des irradiations externes}

La partie supérieure de la tête de mesure est fermée par un badge contenant deux pastilles au fluorure de lithium, dosimètres thermoluminescents qui enregistrent les équivalents de dose dus essentiellement aux rayonnements gamma auxquels sont soumis les travailleurs. La mesure est basée sur la propriété que présentent certains cristaux de libérer l'énergie qu'ils ont emmagasinée lors d'une irradiation sous la forme d'une émission lumineuse qui apparaît quand on les chauffe à une température suffisante $\left(200\right.$ à $\left.300^{\circ} \mathrm{C}\right)$ pour stimuler le phénomène.

C'est le $1^{\mathrm{er}}$ janvier 1986 qu'ont été mis en place les dosimètres thermoluminescents dans les mines d'uranium et les usines de traitement françaises; ils remplacent les dosimètres photographiques (type PS1 du CEA) jusqu'alors utilisés en dosimétrie individuelle et dont la sensibilité (non lecture au-dessous de 0,30 mSv), acceptable pour les expositions significatives, était insuffisante pour la détermination de la majorité des doses rencontrées dans les mines et les usines.

\subsubsection{Laboratoire d'exploitation dosimétrique}

La période normale d'utilisation des dosimètres individuels intégrés est d'un mois. Après cette période de port par le travailleur, période au cours de laquelle les agents de radioprotection mesurent chaque semaine les débits de prélèvement de l'appareil, le service de radioprotection procède au changement des têtes de mesure et la tête ayant servi est envoyée pour traitement au laboratoire d'exploitation dosimétrique qui effectue les opérations suivantes:

- démontage des têtes ; 
- développement du film en nitrate de cellulose, lecture automatique des traces et détermination de l'énergie alpha potentielle des émetteurs alpha à vie courte ;

- comptage alpha des filtres pour déterminer l'activité alpha des émetteurs alpha à vie longue;

- lecture des équivalents de dose dus aux rayonnements gamma.

Chaque système de lecture est géré par microordinateur et un listing, fournissant, pour chaque numéro de tête, les 3 résultats d'exposition, est adressé à un centre de calcul dosimétrique qui, rapprochant le numéro de tête du numéro de dosimétrie du porteur, dresse la fiche individuelle dosimétrique de chaque agent et l'ensemble des états statistiques devant être adressés aux médecins du travail des sociétés et aux administrations concernées.

Le laboratoire d'exploitation dosimétrique actuellement en service permet de traiter 2000 têtes de dosimètres par mois. Quatre personnes travaillent à plein temps au laboratoire et l'ensemble des résultats est fourni 3 semaines environ après la fin de la période d'exposition (si cela s'avère nécessaire pour quelques têtes, un traitement particulier permet de connaître le résultat des expositions 2 ou 3 jours après réception des têtes au laboratoire).

Durant le mois d'exploitation des têtes exposées, le laboratoire doit remonter de nouvelles têtes : après lavage des corps, les têtes sont rechargées avec un nouveau filtre et un nouveau nitrate de cellulose, assemblées avec des collimateurs et des écrans sélecteurs neufs (pour éviter la contamination et des traces parasites sur le détecteur) et avec des dosimètres thermoluminescents remis à zéro. L'ensemble, dûment numéroté et repéré suivant les exploitations, est réexpédié à l'utilisateur.

\subsection{Résultats dosimétriques obtenus}

Les résultats dosimétriques présentés ci-après sont ceux des mines et usines dont le CRPM a assuré la radioprotection en France en 1989 et qui sont exploitées par la Cogéma et la SIMO ${ }^{3}$ sur les trois divisions minières de la Vendée, de La Crouzille et de l'Hérault, par Total Compagnie minière France sur les deux divisions Nord et Sud Massif-Central, par la CFM ${ }^{4)}$ en Corrèze et en Creuse. En 1989, ces sociétés ont extrait environ $2864 \mathrm{t}$ d'uranium dans 12 mines souterraines et 709 t sur 5 sites de mines à ciel ouvert, et les 5 usines de traitement ont enfûté environ 3069 t d'uranium. La teneur moyenne des minerais exploités varie de 0,1 à $0,4 \%$ avec la possibilité de rencontrer ponctuellement des chantiers où la teneur atteint quelques pour cent.

1513 agents des mines souterraines, 412 agents des mines à ciel ouvert et 456 agents des usines de traitement ont fait l'objet d'une surveillance dosimétrique individuelle ou de fonction. La population prise en compte dans les résultats dosimétriques est l'ensemble du personnel exposé sur un siège minier (mineurs à l'abattage, mécaniciens, électriciens, contrôle géologique, maîtrise...) ou dans une usine (agents de fabrication, entretien, maîtrise...).

3) SIMO : Société industrielle des minerais de l'Ouest.

4) CFM : Compagnie française de Mokta. 
La figure 3 présente, pour l'ensemble des mines souterraines et à ciel ouvert et des usines de traitement du minerai, le taux annuel moyen d'exposition totale. En France, en 1989, ce taux varie entre 0,18 et 0,38 pour les travaux miniers souterrains, entre 0,05 et 0,19 pour les mines à ciel ouvert et entre 0,17 et 0,25 dans les usines de traitement du minerai.
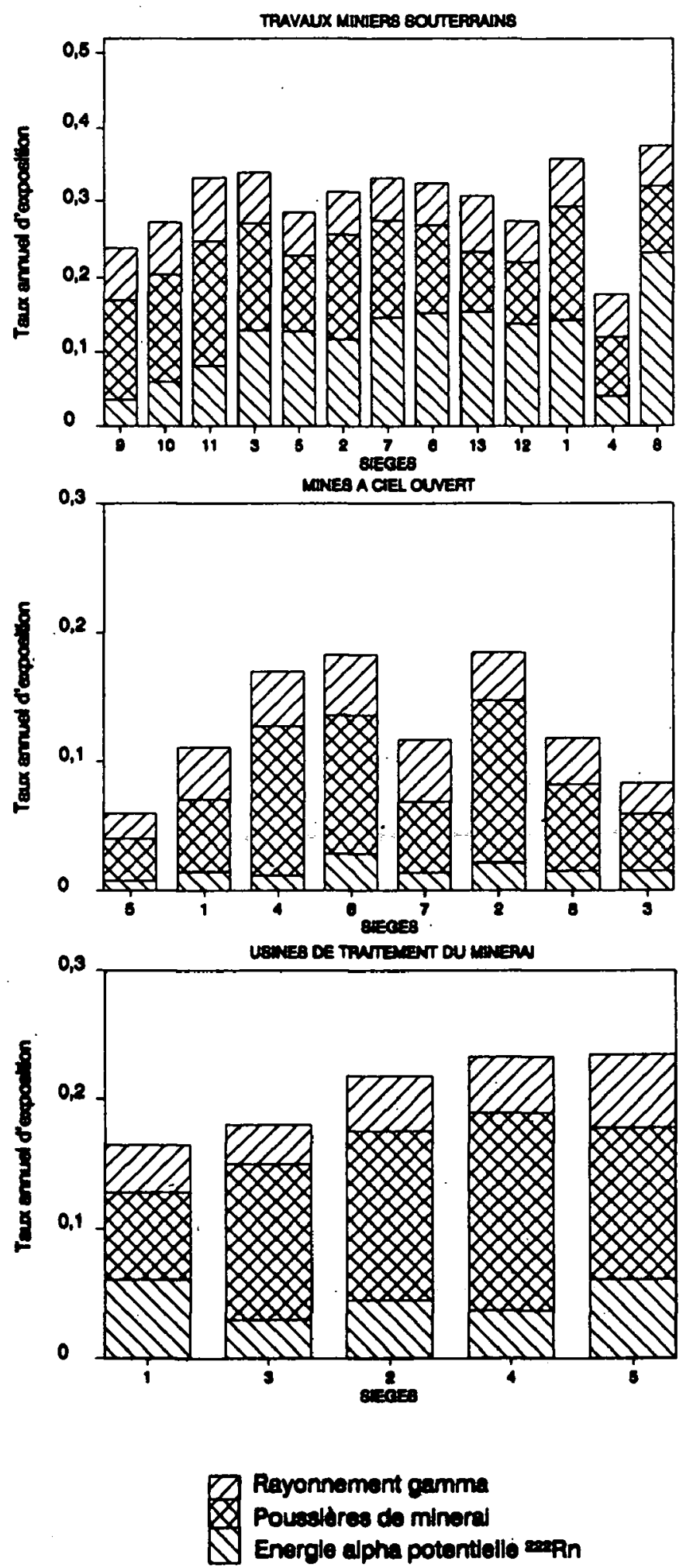

Fig. 3. - Taux annuel moyen d'exposition totale en 1989. 
La CIPR [5], en dehors des recommandations concernant les limites individuelles a précisé, au paragraphe 100 de sa publication $n^{\circ} 26$, que "la répartition des équivalents de dose annuels à l'intérieur de larges groupes professionnels s'est révélée très souvent comme étant conforme à une fonction log-normale avec une moyenne arithmétique d'environ 5 $\mathrm{mSv}$ et avec très peu de valeurs approchant la limite". On constate que cette moyenne arithmétique de $5 \mathrm{mSv} / \mathrm{an}$ peut, sans doute, être atteinte dans les usines de traitement et les mines à ciel ouvert mais, pour l'ensemble du personnel exposé d'un siège minier souterrain, elle ne paraît pas être actuellement un objectif réaliste.

La figure 4 montre que des progrès importants ont été accomplis pour l'ensemble des mines souterraines de l'Ouest et du Centre de la France. La dose efficace moyenne annuelle, égale au taux annuel d'exposition totale multiplié par $50 \mathrm{mSv}$, a été divisée par 2 environ depuis les années 1970 .

MINES SOUTERRAINES DU CENTRE DE LA FRANCE

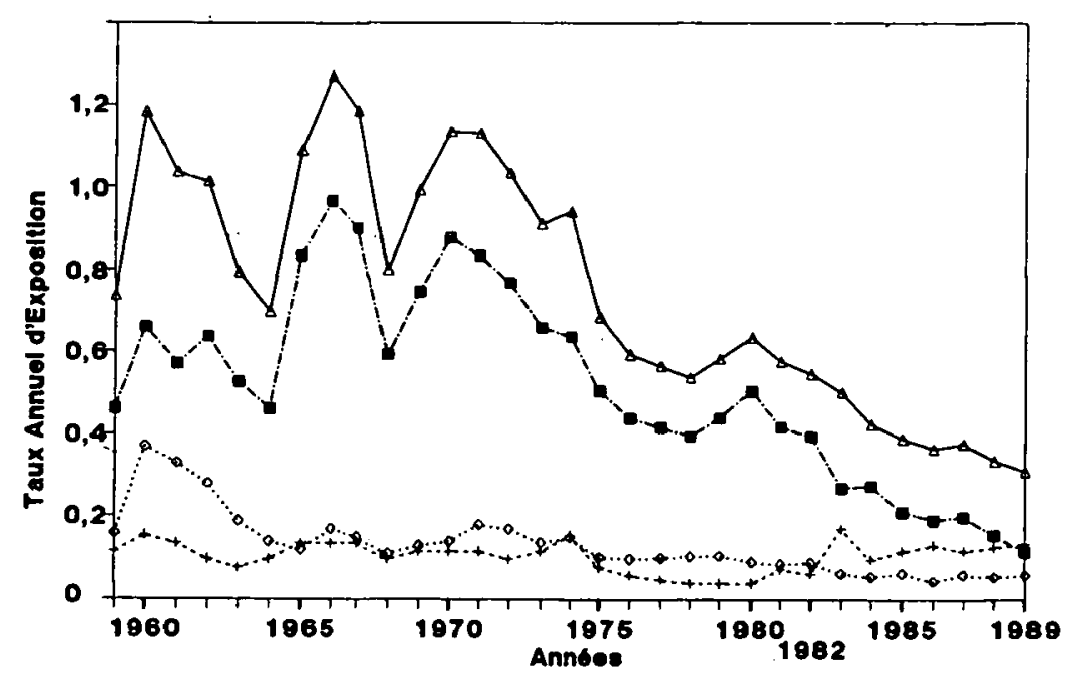

MINES SOUTERRAINES DE L'OUEST DE LA FRANCE

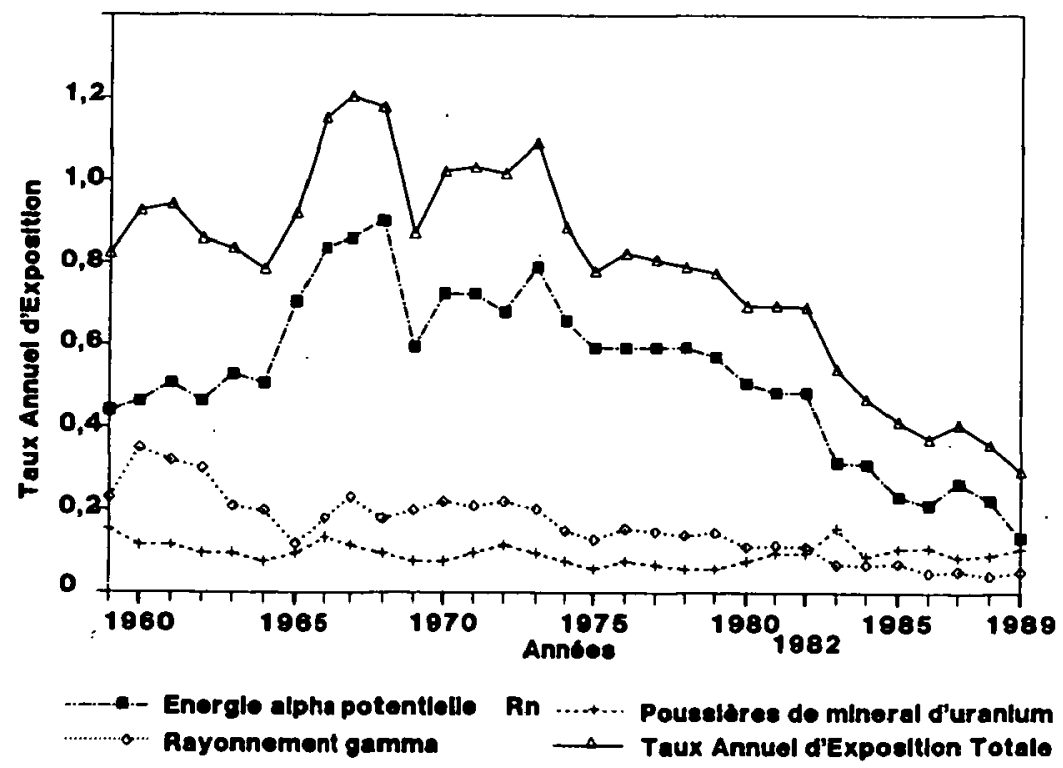

Fig. 4. - Evolution des taux d'exposition moyens annuels du personnel "fond" des mines (1982 : passage a la dosimétrle indivlduelle intégrée). 
La figure 5 présente la répartition des doses individuelles annuelles reçues par le personnel. En 1989, personne n'atteint le taux annuel d'exposition totale de 1 ; cependant, le risque de dépassement des limites individuelles sur-une période de 12 mois roulants reste élevé dans les mines souterraines. Le respect de la réglementation française nécessite encore une grande vigilance de la part des agents de radioprotection et un effort constant des exploitants.
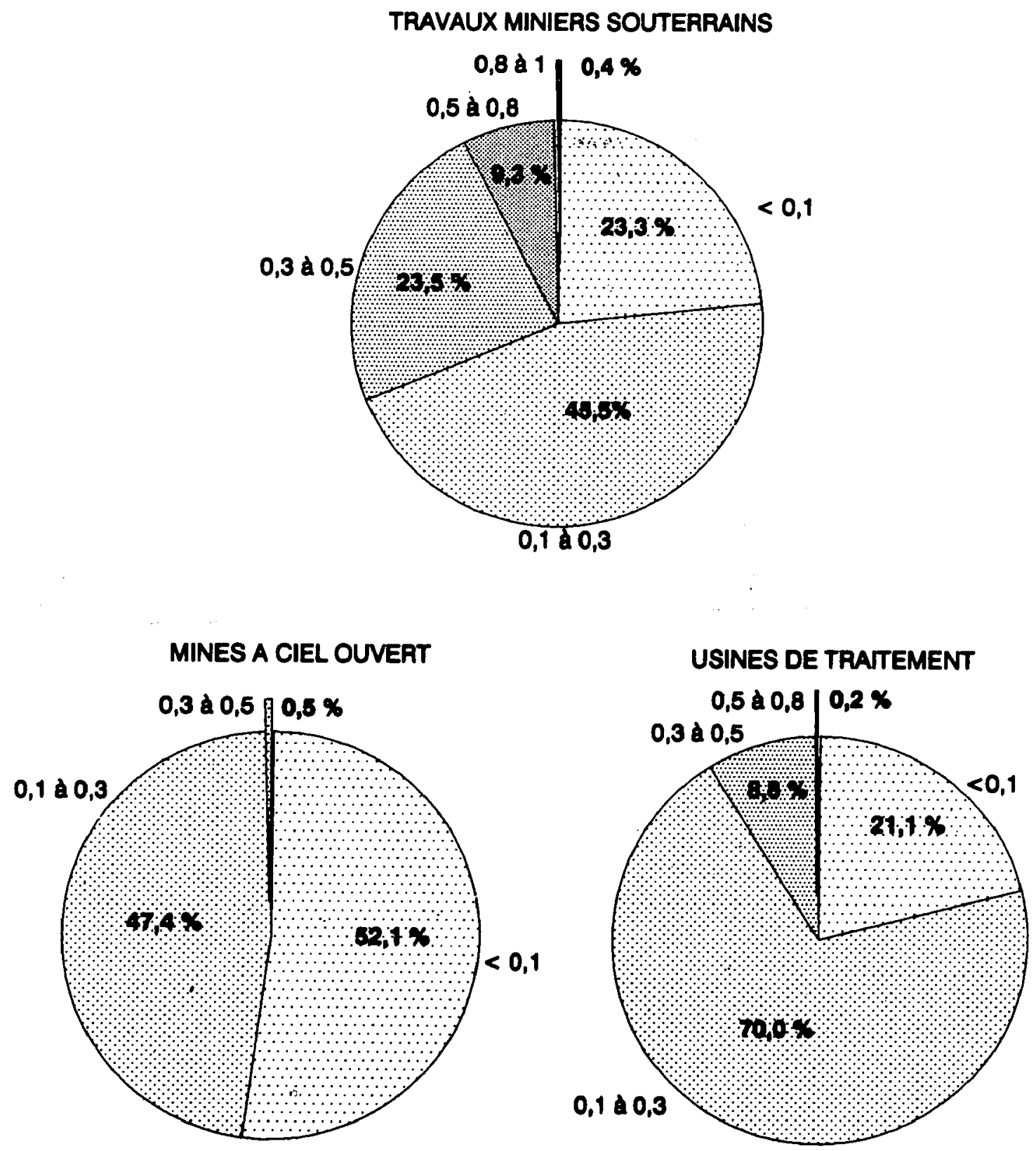

Flg. 5. - Répartition de l'effectlf TMS, MCO et usines françalses en fonction de leur taux annuel d'exposition totale (TAET)

TAETA $=\frac{\text { Gamma }}{50 \mathrm{mSv}}+\frac{\text { EAP }^{222} \mathrm{Rn}}{20 \mathrm{~mJ}}+\frac{\text { Poussières de mineral }}{1700 \mathrm{~Bq} \alpha}+\frac{\text { Poussières d'uranate }}{30000 \mathrm{~Bq} \alpha}$ 
Si l'on rapproche les données radiologiques de chaque exploitation (doses collectives) avec des données d'exploitation (production de minerai et d'uranium, productivité d'extraction du minerai, puissance de ventilation primaire et secondaire), on peut définir des indices qui permettent de comparer entre elles les qualités de protection des différentes exploitations. On définit ainsi l'irradiation spécifique comme le rapport de la dose collective dans le risque considéré au nombre de tonnes d'uranium extraites ou enfûtées. En France, si l'on considère la dose collective totale due aux 3 risques gamma, radon, poussières, cet indice varie en 1989 (fig. 6) :

- de 3 à 27 p.mSv. ${ }^{-1}$ (personne-millisievert par tonne d'uranium extraite) dans les mines souterraines;

- de 1,1 à 3,2 p.mSv. $t_{u}^{-1}$ dans les mines à ciel ouvert;

- de 0,9 à 7,5 p.mSv.t ${ }_{u}^{-1}$ dans les usines de traitement.
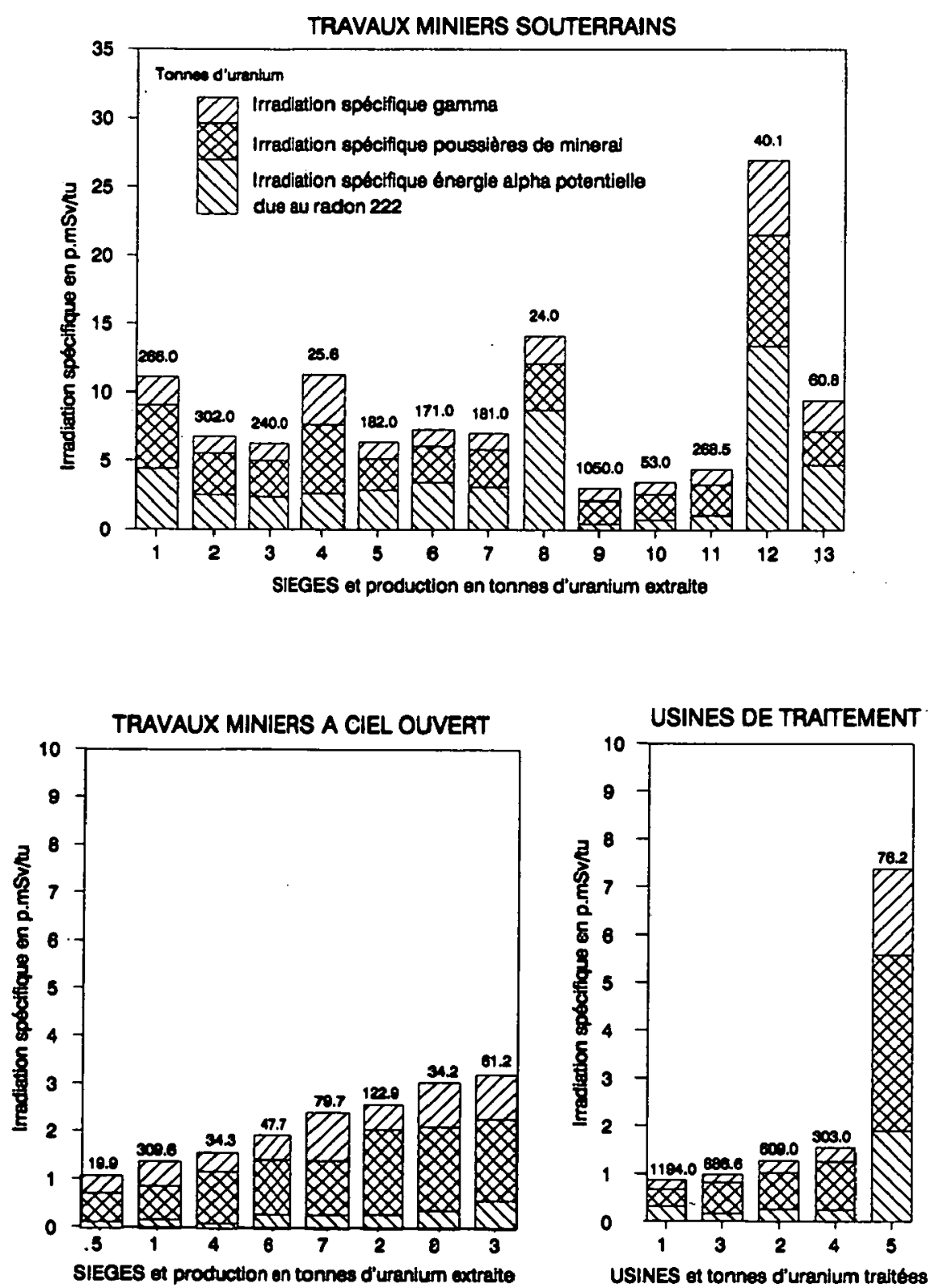

Fig. 6. - Irradlations spécifiques totales en 1989 
Dans les mines souterraines, si l'on rapporte la dose collective "EAP ${ }^{222} \mathrm{Rn}$ " à la puissance totale d'aérage installée (aérage primaire + aérage secondaire), on obtient un indice dont l'évolution témoigne de l'utilisation des systèmes de ventilation mis en place. Pour les mines souterraines françaises, en 1989, cet indice varie de 0,2 p.mSv.kW-1 à 6,6 p.mSv.kW ${ }^{-1}$, la puissance totale d'aérage installée variant de $0,8 \mathrm{~kW}$ à $6,7 \mathrm{~kW}$ par tonne d'uranium extraite.

La diminution de l'ensemble des indices est le but poursuivi : elle est liée d'une part à l'amélioration de la productivité des installations et, d'autre part aux actions de prévention menées par les agents de radioprotection.

\section{PRÉVENTION DES RISQUES RADIOLOGIQUES}

C'est dans les années 1970 que la doctrine de la CIPR s'est particulièrement développée autour du principe d'optimisation de la protection. Ce principe paraissait être en parfaite continuité avec la politique jusqu'alors développée pour la radioprotection des mines d'uranium et fut donc adopté sans restriction. Maintenant repris par l'article $11 \mathrm{du}$ décret $n^{\circ}$ 89-502 du 13 juillet 1989 complétant le règlement général des industries extractives, il continue à être appliqué avec efficacité sur l'ensemble des installations françaises.

Tout d'abord, il est nécessaire de rappeler que les actions de prévention liées à la politique d'optimisation de la radioprotection, maintenant adoptée par l'ensemble des exploitants français, dépendent en grande partie de la méthode d'exploitation choisie. Avant l'ouverture d'un siège minier, c'est ce choix qui engage de façon déterminante ce que sera le niveau des expositions subies par les travailleurs de cette exploitation. Cette prise en compte des critères radiologiques à côté des critères technico-économiques doit également apparaître tout au long de la vie de la mine, au moment des décisions nouvelles devant être prises par l'exploitant.

Si l'on suppose que cette première étape de recherche de l'optimum au moment de la sélection des méthodes d'exploitation est menée, la politique d'optimisation de la radioprotection impose une deuxième étape qui consiste à maintenir des conditions optimales vis-à-vis des risques radiologiques pendant la durée de l'exploitation. C'est dans le cadre de cette deuxième étape que l'action des services de radioprotection doit se concrétiser chaque jour dans les chantiers.

En 1983, l'introduction de la dosimétrie alpha individuelle dans les mines françaises en remplacement de la dosimétrie d'ambiance pratiquée depuis 1955 a été un changement fondamental dans la manière de concevoir et d'organiser le travail des équipes de surveillance radiologique. Les fonctions de comptabilisation des doses individuelles et celles qui permettent de remplir les tâches de prévention sont désormais complètement dissociées : les doses individuelles sont fournies directement 
par les dosimètres portés par les agents, ces appareils ne demandant chaque mois que quelques heures de maintenance et de contrôle. Ainsi, les agents des équipes de radioprotection peuvent consacrer l'essentiel de leur temps aux actions de prévention.

Des procédures de collecte de données sur les ambiances physiques sont établies en vue de déterminer les emplacements et l'intensité des sources d'irradiation (liées en particulier au radon 222 pour les mines souterraines ou aux poussières de minerai dans les mines à ciel ouvert et les usines de traitement) et de vérifier que les moyens de prévention des risques mis en œuvre, en particulier ceux qui permettent l'assainissement radioactif de l'atmosphère, sont utilisés au mieux de leurs performances propres et selon l'état de l'air, et qu'ils restent adaptés aux sources d'exposition et aux configurations des postes de travail. La fréquence des mesures est fonction des niveaux rencontrés et peut varier d'une zone de travail à une autre. Elle est généralement hebdomadaire dans les mines et les usines françaises mais devient quotidienne lorsque les niveaux d'exposition aux rayonnements et de contamination radioactive de l'air dépassent les niveaux opérationnels fixés dans la réglementation ou introduits comme objectifs à atteindre par l'exploitant. Les objectifs les plus courants sont, pour les mines françaises:

- $25 \mu \mathrm{Gy} \cdot \mathrm{h}^{-1}$ pour les rayonnements gamma,

$-8,3 \mu \mathrm{J} \cdot \mathrm{m}^{-3}$ pour l'énergie alpha potentielle,

- $750 \mathrm{mBq}$ alpha. $\mathrm{m}^{-3}$ pour les poussières de minerai,

- $5500 \mathrm{~Bq}$ alpha. $\mathrm{m}^{-3}$ pour le radon 222.

Les agents de radioprotection contrôlent régulièrement les concentrations en radon 222 (utilisation de fioles scintillantes), les concentrations en énergie alpha potentielle due aux descendants à vie courte du radon 222 (utilisation de l'appareil type "MIMIL" mis au point par le CRPM), les concentrations en poussières radioactives (utilisation d'appareils autonomes de prélèvements sur filtres) et les débits de dose dus aux rayonnements gamma (utilisation de radiamètres). La collecte de ces mesures radiologiques est complétée par les données caractérisant les lieux de travail. En particulier, dans les mines souterraines, les données géométriques des chantiers et les caractéristiques de l'aérage primaire et secondaire sont répertoriées. Dans les mines à ciel ouvert et les usines de traitement, l'exploitation mensuelle des niveaux moyens mesurés par les dosimètres de fonction permettent également d'orienter les actions de prévention.

Le tableau I fournit les résultats moyens de la surveillance des ambiances physiques pour l'année 1989. Un nombre très important de données est ainsi recueilli et une exploitation systématique permet:

- de mettre en évidence, en collaboration étroite avec les exploitants, les situations anormales, d'en identifier les causes et de choisir les solutions optimales à mettre en cuvre pour améliorer la qualité des ambiances de travail; 
TABLEAU I

Contrôles des ambiances physiques - Résultats moyens annuels 1989

\begin{tabular}{|c|c|c|c|}
\hline \multirow[b]{2}{*}{$\begin{array}{l}\text { Mines } \\
\text { souterraines * }\end{array}$} & \multicolumn{3}{|c|}{ Concentrations moyennes dans les chantiers } \\
\hline & $\begin{array}{c}\text { Radon } 222 \\
\text { Bq. }^{-3}\end{array}$ & $\begin{array}{c}\text { EAP }{ }^{222} \mathrm{Rn} \\
\mu \mathrm{J} \cdot \mathrm{m}^{-3}\end{array}$ & $\begin{array}{l}\text { Poussières } \\
\text { de minerai } \\
\text { mBq alpha.m }\end{array}$ \\
\hline 1 & 3968 & 10 & 64 \\
\hline 2 & 2112 & 3,2 & 49 \\
\hline 3 & 1536 & $3, \underline{6}$ & 70 \\
\hline 4 & 1116 & 2,9 & 43 \\
\hline 5 & 2550 & 4,2 & 50 \\
\hline 6 & 3035 & 5,0 & 79 \\
\hline 7 & 6320 & 8,1 & 72 \\
\hline 8 & 8535 & 6,9 & 22 \\
\hline 9 & 1060 & 1,1 & 187 \\
\hline 10 & 1320 & 0,9 & 21 \\
\hline 11 & 3173 & 3,2 & 46 \\
\hline 12 & 2030 & 5,0 & 98 \\
\hline
\end{tabular}

\begin{tabular}{|c|c|c|c|}
\hline \multirow[b]{2}{*}{$\begin{array}{l}\text { Mines } \\
\text { à ciel ouvert ** }\end{array}$} & \multicolumn{3}{|c|}{ Concentrations moyennes par fonction } \\
\hline & $\begin{array}{c}\text { EAP }{ }^{222} \mathrm{Rn} \\
\mu \mathrm{J} \cdot \mathrm{m}^{-3}\end{array}$ & $\begin{array}{c}\text { Poussières } \\
\text { de } \\
\text { minerai } \\
\text { mBq.m }\end{array}$ & $\begin{array}{c}\text { Débit de dose moyen } \\
\text { en irradiation } \\
\text { externe } \\
\mu G y . h^{-1}\end{array}$ \\
\hline Foration & 0,56 & 149 & 2,6 \\
\hline Chargement & 0,28 & 120 & 2,2 \\
\hline Roulage & 0,18 & 108 & 1,7 \\
\hline Echantillonnage & 0,41 & 162 & 2,9 \\
\hline
\end{tabular}

\begin{tabular}{|c|c|c|c|c|}
\hline \multirow[b]{2}{*}{$\begin{array}{c}\text { Usines } \\
\text { de traitement }\end{array}$} & \multicolumn{3}{|c|}{ Concentrations moyennes par fonction } & \multirow{2}{*}{$\begin{array}{l}\text { Débit de dose } \\
\text { en irradiation } \\
\text { externe } \\
\mu \mathrm{Gy} \cdot \mathrm{h}^{-1}\end{array}$} \\
\hline & $\begin{array}{l}\underset{\text { EAP }}{222} \mathrm{Rn} \\
\mu \mathrm{J} \cdot \mathrm{m}^{-3}\end{array}$ & $\begin{array}{l}\text { Poussières de } \\
\text { minerai } \\
\text { mBq alpha. } \mathbf{m}^{-3}\end{array}$ & $\begin{array}{l}\text { Poussières } \\
\text { d'uranate } \\
\text { mBq alpha.m-3 }\end{array}$ & \\
\hline $\begin{array}{l}\text { Préparation mécanique } \\
\text { du minerai }\end{array}$ & 0,87 & 167 & & 1,9 \\
\hline $\begin{array}{l}\text { Fabrication (filtration, } \\
\text { attaque }\end{array}$ & 0,49 & 132 & 255 & 1,5 \\
\hline Maintenance & 0,46 & 141 & & 1,4 \\
\hline Echantillonnage minerai & 0,51 & 151 & & 1,8 \\
\hline Enfûtage-échant. uranate & & & 2954 & \\
\hline
\end{tabular}

- Valeurs moyennes calculées à partir des contrôles ponctuels effectués par les agents de radioprotection.

** Valeurs moyennes calculées à partir de 85 valeurs mensuelles obtenues avec les dosimètres de fonction portés dans 10 mines à ciel ouvert françaises.

*** Valeurs moyennes calculées à partir de 1030 valeurs mensuelles obtenues avec les dosimètres de fonction portés dans 4 usines de traitement. 
- de suivre, à moyen terme, l'évolution d'un certain nombre de paramètres concernant les sources de polluants, l'efficacité des moyens de prévention mis en œuvre ainsi que les coûts afin de permettre la mise en œuvre du principe d'optimisation [7].

La présence régulière d'un agent de radioprotection dans les zones de travail est nécessaire pour inciter le personnel exploitant à veiller à la mise en œuvre des moyens classiques de limitation des expositions: foration à l'eau, arrosage des produits et des parements après les tirs et au chargement, bonne installation des canalisations d'aérage secondaire, changement des filtres équipant les ventilateurs d'aérage secondaire insonorisés, retour dans le chantier $30 \mathrm{~min}$ au moins après le tir...

Cette action de tous les jours permet ainsi d'obtenir de bonnes performances radiologiques et surtout de les maintenir. Leur amélioration nécessite, cependant, de nouvelles options techniques de prévention, par exemple :

- pour le risque dû au radon 222 et à ses descendants : contrôle du transport convectif du radon 222 dans les roches encaissantes (organisation d'un drainage des nappes de radon; mise en dépression des vieux travaux; mise en pression des ouvrages en exploitation);

- pour les irradiations externes, dans le cas de gisements à forte teneur: blindage des engins; organisation de l'exploitation visant à limiter les sources d'irradiation (remblayages bétonnés); l'éloignement des agents par rapport aux sources est un moyen d'action efficace et, dans ce sens, la mécanisation des chantiers dans les mines ou l'automatisation des usines sont à considérer.

La mise en œuvre opérationnelle de toutes ces actions de prévention ne peut, cependant, être amorcée qu'après des études et des recherches sur la réduction des risques radiologiques qui, depuis de longues années, ont été supportées par le Département de protection technique de I'IPSN.

Cet effort de recherche de base porte essentiellement sur les problèmes posés par l'assainissement de l'atmosphère des chantiers. Ce sont :

- des recherches sur les mécanismes de la pollution radioactive de l'atmosphère avec l'étude de l'origine de la composante radioactive de la pollution de l'air et l'étude de la granulométrie de l'aérosol radioactif ;

- des recherches sur les moyens techniques de l'assainissement des chantiers ;

- des recherches sur la modélisation des processus d'exposition des mineurs ;

- des recherches sur l'instrumentation en radioprotection dans les mines d'uranium (appareils de contrôles de prévention et amélioration des appareils de dosimétrie). 


\section{RÉFÉRENCES}

[1] BERNHARD S., LE GAC J., ZETTWOOG P., SEGUIN H. - Identification of non uranium mines which present a radon risk through measurements of exhaust air. In : Occupational radiation safety in mining, Toronto (Canada), 14-18 Oct. 1984. (H. Stocker, Ed.). Toronto : Canadian Nuclear Association, 1985, Vol. 1, 307-316.

[2] BERHNARD S., LE GAC J., ZETTWOOG P., SEGUIN H. - Occupational exposure to $R n$ in non-uranium mines in the European community. Health Phys., 1985, 49 (5) 999-1002.

[3] BERNHARD S., PINEAU J.F., RANNOU A., ZETTWOOG P. - 1983 : one year of individual dosimetry in french mines. In : Occupational radiation safety in mining, Toronto (Canada), 14-18 Oct. 1984. (H. Stocker, Ed.). Toronto : Canadian Nuclear Association, 1985, Vol. 2, 526-540.

[4] BERNHARD S., KRAEMER G., LE GAC J., ZETTWOOG P. - Organisation et résultats de la surveillance radiologique des personnels des mines d'uranium en France. In : Comité technique AlEA sur les aspects technologiques de l'extraction de l'uranium, Montpellier, 21-24 septembre 1987.

[5] COMMISSION INTERNATIONALE DE PROTECTION RADIOLOGIQUE (CIPR) Recommandations de la CIPR (adoptées le 17 janvier 1977). (Publication CIPR 26). Oxford: Pergamon Press, 1978.

[6] FOURCADE N., ZETTWOOG P. - A dynamic approach to the implementation of the optimization principles in uraium mines. In : Occupational radiation safety in mining, Toronto (Canada), 14-18 Oct. 1984. (H. Stocker, Ed.). Toronto : Canadian nuclear association, 1985, Vol. 1, 57-71.

[7] KRAEMER G., ZETTWOOG P. - Nouveaux développements de la mise en œuvre du principe d'optimisation dans les mines d'uranium. In : Optimization of radiation protection, AIEA/AEN Vienna, 10-14 March 1986. Vienne: AIEA, 1986, 273-298.

[8] PRADEL J., ZETTWOOG P. - Hier et maintenant : la radioprotection dans les mines d'uranium. Rev. Gén. Nucl., 1984, $n^{\circ} 1,38-57$.

[9] SZCHMITZ J., PINEAU J.F., ZETTWOOG P. - Experiences with diferrent techniques for routine dose assessment of uranium miners. In : IRPA 4. European congress, twenty years experience in radiation protection - a review and outlook, extended synopses. Salzburg, 15-19 sept. 1986. Seibersdorf : Osterreichischer Verlag für Strahlenschutz, 1988, p. 65.

[10] ZETTWOOG P. - Réflexions sur la mise en œuvre des réglementations en matière de limitation des risques radiologiques dans les mines d'uranium. Etude du cas du Niger et du cas de la France. In : International workshop on radiological protection in mining, Darwin (Australie), 4-8 April 1988 (J. KVASNICKA, Ed.) volume $A, \mathbf{2 2 8 - 3 0 0 .}$ 